\title{
Antimicrobial activity of some synthesized glucopyranosyl-pyrimidine carbonitrile and fused pyrimidine systems
}

\author{
HAYAM H. SAYED ${ }^{1}$ \\ HEBAT-ALLAH S. ABBAS ${ }^{1}$ \\ EMAN M. H. MORSI ${ }^{1}$ \\ ABD EL-GALIL E. AMR ${ }^{2, *}$ \\ NAYERA A. M. ABDELWAHAD ${ }^{3}$ \\ 1 Photochemistry Department \\ National Research Centre \\ 12622 Dokki, Cairo, Egypt \\ 2 Department of Pharmaceutical \\ Chemistry, College of Pharmacy \\ King Saud University, Riyadh 11451 \\ Saudi Arabia \\ ${ }^{3}$ Chemistry of Natural and Microbial \\ Product Department, National Research \\ Centre, 12622 Dokki, Cairo, Egypt
}

\begin{abstract}
3-Amino-5-(4-chlorophenylamino)-4-cyanofuran-2-carboxamide (2) was used as the key molecule for preparation of various furopyrimidines 3-9 and formation of spiro-cycloalkane furopyrimidines 10, 11. Also, poly fused heterocyclic compounds 13-17 were prepared from compound 2. The synthesized compounds were screened for their antimicrobial activity.
\end{abstract}

Keywords: pyrimidine, glucopyranosyl-pyrimidine, oxazine, antimicrobial activity

Accepted October 4, 2010

This research is an extension of our studies on pyrimidine and pyrimidinethione derivatives as synthons to prepare fused heterocyclic compounds $(1,2)$. Some of the pyrimidine and fused heterocyclic pyrimidine derivatives have proved to be active antiviral, antitumor, analgesic and antimicrobial agents (3-6). In addition, pyrimidothiazine, thiazolopyrimidine and oxazolidinone derivatives are of great antimicrobial activity (7). Recently, we have found that certain substituted pyrimidines and their heterocyclic derivatives show antimicrobial and antiinflammatory $(8,9)$ as well as antitumor activities $(10,11)$. On the other hand, thioxopyrimidine and thiazolopyrimidine derivatives have anticancer activities $(12,13)$. In view of these observations and in continuation of our previous work in heterocyclic chemistry, we have synthesized some new pyrimidine and thiazolopyrimidine derivatives and tested their antimicrobial activity.

\footnotetext{
*Correspondence; e-mail: aamr1963@yahoo.com
} 
H. H. Sayed et al.: Antimicrobial activity of some synthesized glucopyranosyl-pyrimidine carbonitrile and fused pyrimidine systems, Acta Pharm. 60 (2010) 479-491.

\section{EXPERIMENTAL}

All melting points are uncorrected and were measured using an Electro-thermal IA 9100 apparatus (Shimadzu, Japan). IR spectra were recorded as potassium bromide pellets on a Perkin-Elmer 1650 spectrophotometer (Perkin-Elmer, USA). ${ }^{1} \mathrm{H}$ NMR was recorded on a Jeol-Ex-270 NMR spectrometer (Jeol, Japan) and chemical shifts were expressed as part per million (ppm $\delta$ values) against TMS as the internal reference standard. Mass spectra were recorded on a VG 2AM-3F mass spectrometer (Thermo Electron, USA). Microanalyses were operated using a Yanaco CHN Corder Elemental Analyzer (Japan) and the results were within the accepted range $( \pm 0.2 \%)$ of calculated values. Follow-up of the reactions and purity checking of the compounds were done by TLC on silica gel-coated aluminum sheets (Type 60 F254, Merck, Germany).

\section{Syntheses}

2-[3-(4-Chlorophenyl)-4-oxooxazolidin-2-ylidene]malononitrile (1). - To a stirred mixture of malononitrile $(0.66 \mathrm{~g}, 0.01 \mathrm{~mol}), p$-chlorophenylisocyanate $(1.53 \mathrm{~g}, 0.01 \mathrm{~mol})$ in distilled water $(20 \mathrm{~mL})$ containing $\mathrm{KOH}(1 \mathrm{~g})$ at room temperature, ethyl chloroacetate $(1.22 \mathrm{~g}$, $0.01 \mathrm{~mol})$ in ethanol $(25 \mathrm{~mL})$ was added after $1 \mathrm{~h}$. The reaction mixture was heated under reflux for $2 \mathrm{~h}$, the obtained solid was collected by filtration and crystallized from methanol to give compound $\mathbf{1}$ as white crystals.

3-Amino-5-(4-chlorophenylamino)-4-cyanofuran-2-carboxamide (2). - A solution of compound $1(2.56 \mathrm{~g}, 0.01 \mathrm{~mol})$ in ammonia solution $(25 \%, 30 \mathrm{~mL})$ was stirred at room temperature for $6 \mathrm{~h}$. The solid formed was filtered off, dried and crystallized from chloroform to give compound 2 as a brown powder.

6-(4-Chlorophenylamino)-4-oxo-3,4-dihydrofuro[3,2-d]pyrimidin-7-carboxamide (3). - A mixture of compound $2(2.77 \mathrm{~g}, 0.01 \mathrm{~mol})$ and formic acid $(20 \mathrm{~mL})$ was refluxed for $6 \mathrm{~h}$. The reaction mixture was concentrated under reduced pressure, the obtained precipitate was collected by filtration, dried and crystallized from dioxane to give compound 3 as yellow crystals.

6-(4-Chlorophenylamino)-4-oxo-3-phenyl-2-thioxo-1,2,3,4-tetrahydro-furo[3,2-d]pyrimidine-7-carbonitrile (4). - A mixture of compound $2(2.77 \mathrm{~g}, 0.01 \mathrm{~mol})$ and phenylisothiocyanate $(1.35 \mathrm{~g}, 0.01 \mathrm{~mol})$ in glacial acetic acid $(25 \mathrm{~mL})$ was refluxed for $6 \mathrm{~h}$. The reaction mixture was allowed to cool; the formed solid was filtered off, dried and crystallized from ethyl acetate to give 4 as a light brown powder.

6-(4-Chlorophenylamino)-2,4-dioxo-1,2,3,4-tetrahydro-furo[3,2-d]pyrimidine-7-carbonitrile (5). - A mixture of compound $2(2.77 \mathrm{~g}, 0.01 \mathrm{~mol})$ and ethylchloroformate $(1.08 \mathrm{~g}, 0.01$ $\mathrm{mol})$ in acetic acid $(15 \mathrm{~mL})$ was refluxed for $10 \mathrm{~h}$. The reaction mixture was allowed to cool, the solid product was filtered off and crystallized from DMSO to give the corresponding compound 5 as a brown powder.

1-(3-Chloro-2-oxopropyl)-6-(4-chlorophenylamino)-4-oxo-3-phenyl-2-thioxo-1,2,3,4-tetrahydro-furo[3,2-d]pyrimidine-7-carbonitrile (6) and 6-(4-chlorophenylamino)-1-(oxiran-2-yl-methyl)-4-oxo-3-phenyl-2-thioxo-1,2,3,4-tetrahydro-furo[3,2-d]pyrimidine-7-carbonitrile (7). - A mixture of compound $4(3.95 \mathrm{~g}, 0.01 \mathrm{~mol})$ and sodium hydride $(0.24 \mathrm{~g}, 0.01 \mathrm{~mol})$ in dry 
H. H. Sayed et al.: Antimicrobial activity of some synthesized glucopyranosyl-pyrimidine carbonitrile and fused pyrimidine systems, Acta Pharm. 60 (2010) 479-491.

dioxane $(20 \mathrm{~mL})$ was stirred at $60^{\circ} \mathrm{C}$ for $3 \mathrm{~h}$. The reaction mixture was cooled at room temperature, then dichloroacetone or epichlorohydrine $(0.01 \mathrm{~mol})$ was added under stirring. Stirring was continued for $5 \mathrm{~h}$ at room temperature. The reaction mixture was evaporated under reduced pressure; the residue was washed with distilled water, filtered off and crystallized from methanol to give compound 6 or 7.

6-(4-Chlorophenylamino)-4-oxo-3-phenyl-2-(2',3',4',6'-tetra-O-acetyl-1'-thio- $\beta$-D-glucopyranosyl)-1,2,3,4-tetrahydrofuro[3,2-d]pyrimidine-7-carbonitrile (8). - To a solution of 2 (2.77 $\mathrm{g}, 0.01 \mathrm{~mol})$ in aqueous potassium hydroxide $(0.56 \mathrm{~g}, 0.01 \mathrm{~mol})$ in distilled water $(5 \mathrm{~mL})$, a solution of 2,3,4,6-tetra- $O$-acetyl- $\alpha$-D-glucopyranosyl bromide $(4.11 \mathrm{~g}, 0.011 \mathrm{~mol})$ in acetone $(30 \mathrm{~mL})$ was added. The reaction mixture was stirred at room temperature for $14 \mathrm{~h}$ (under TLC control). The solvent was evaporated under reduced pressure at $40{ }^{\circ} \mathrm{C}$ and the crude product was filtered off and washed with distilled water to remove $\mathrm{KBr}$ formed. The product was dried, and crystallized from diethyl ether as a brown powder.

6-(4-Chlorophenylamino)-4-oxo-2-phenyl-1,2,3,4-tetrahydrofuro[3,2-d]pyrimidine-7-carbonitrile (9). - A mixture of compound $2(2.77 \mathrm{~g}, 0.01 \mathrm{~mol})$ and benzaldehyde $(1.06 \mathrm{~g}, 0.01$ $\mathrm{mol})$ in glacial acetic acid $(20 \mathrm{~mL})$ was refluxed for $4 \mathrm{~h}$. The reaction mixture was evaporated under reduced pressure and the obtained residue was triturated with diethyl ether. The formed solid was collected by filtration, dried and crystallized from dioxane to give compound 9 as yellow crystals.

6-(4-Chlorophenylamino)-7-cyano-1,3,4-trihydrospirocyclopentane (1',2)-furo[3,2-d]pyrimidin4-one (10) and 6-(4-chlorophenylamino)-7-cyano-1,3,4-trihydrospirocyclohexane(1,2)furo[3,2-d Jpyrimidin-4-one (11). - A mixture of compound 2 (2.77 g, $0.01 \mathrm{~mol})$, cyclopentanone or cyclohexanone $(0.01 \mathrm{~mol})$ and sodium acetate $(2 \mathrm{~g})$ in glacial acetic acid $(20 \mathrm{~mL})$ was refluxed for $6 \mathrm{~h}$. The reaction mixture was cooled, then poured into ice-water. The precipitated solid was filtered off, washed with water, dried and crystallized from ethanol to give compound $\mathbf{1 0}$ or $\mathbf{1 1}$.

6-(4-Chlorophenyl)-6-(7-cyano-2-methyl-4-oxo-4H-furo[3,2-d][1,3]oxazin-6-yl)acetamide (12). - A solution of compound $2(2.77 \mathrm{~g}, 0.01 \mathrm{~mol})$ in acetic anhydride $(20 \mathrm{~mL})$ was refluxed for $8 \mathrm{~h}$. The reaction mixture was concentrated under reduced pressure. The precipitated solid was collected by filtration, dried and crystallized from ethanol to give compound 12 as orange crystals.

1-(4-Chlorophenyl)-4-imino-2,6-dithioxo-1,4,6,7-tetrahydropyrimido[5,4-b]furo[5,4-d]-1,3thiazin-8-one (13). - A mixture of compound 2 (2.77 g, $0.01 \mathrm{~mol})$, carbon disulfide (1.56 g, $0.02 \mathrm{~mol})$ and dry pyridine $(20 \mathrm{~mL})$ was refluxed for $20 \mathrm{~h}$. The reaction mixture was cooled then poured into ice-water. The precipitated solid was filtered off, washed with water, dried and crystallized from DMF.

1-(4-Chlorophenyl)-2,3-dithioxo-8-oxo-2,2',10,11-tetrahydrothiazolo[1,3-b]pyrimido[1,3-el]furo-[5,4-e]pyrimidine (14) and 1-(4-chlorophenyl)-2,3-dithioxo-8-oxo-2,2',3,3',11,12-hexahydro-thiazino[1,3-b]-pyrimido[1,3-e]furo[5,4-e]pyrimidine (15). - A mixture of compound 13 (3.95 g, $0.01 \mathrm{~mol})$, chloroacetic acid or chloropropionic acid $(0.01 \mathrm{~mol})$ and anhydrous sodium acetate $(2 \mathrm{~g})$ was refluxed in glacial acetic acid/acetic anhydride $(40 \mathrm{~mL}, 3: 1)$ for $3 \mathrm{~h}$. The reaction mixture was cooled and poured into water; the obtained precipitate was filtered off and crystallized from methanol to give compound 14 or 15. 
H. H. Sayed et al.: Antimicrobial activity of some synthesized glucopyranosyl-pyrimidine carbonitrile and fused pyrimidine systems, Acta Pharm. 60 (2010) 479-491.

7-[Phenylmethylene]-1-(4-chlorophenyl)-2,3-dithioxo-8-oxo-2,2',10,11-tetrahydrothiazolo[1,3-b]-pyrimido[1,3-e]furo-[5,4-e]pyrimidine (16) and 8-[phenylmethylene]-1-(4-chlorophenyl)-2,3-dithioxo-8-oxo-2,2',3,3',11,12-hexahydrothiazino[1,3-b]-pyrimido[1,3-e]furo[5,4-e]pyrimidine (17). - A mixture of compound 14 or $15(0.01 \mathrm{~mol})$, benzaldehyde $(1.06 \mathrm{~g}, 0.01 \mathrm{~mol})$ and anhydrous sodium acetate $(2 \mathrm{~g})$ in glacial acetic acid/acetic anhydride $(40 \mathrm{~mL}, 3: 1)$ was

Table I. Physical and analytical data of new compounds

\begin{tabular}{|c|c|c|c|c|c|c|c|}
\hline \multirow{2}{*}{$\begin{array}{l}\text { Compd. } \\
\text { No. }\end{array}$} & \multirow{2}{*}{ Formula $\left(M_{\mathrm{r}}\right)$} & \multirow{2}{*}{$\begin{array}{l}\text { M.p. } \\
\left({ }^{\circ} \mathrm{C}\right)\end{array}$} & \multirow{2}{*}{$\begin{array}{c}\text { Yield } \\
(\%)\end{array}$} & \multicolumn{4}{|c|}{ Analysis (\%) (calcd./found) } \\
\hline & & & & $\mathrm{C}$ & $\mathrm{H}$ & $\mathrm{N}$ & $\mathrm{Cl}$ \\
\hline \multirow{2}{*}{1} & \multirow{2}{*}{$\begin{array}{c}\mathrm{C}_{12} \mathrm{H}_{6} \mathrm{ClN}_{3} \mathrm{O}_{2} \\
(259.65)\end{array}$} & \multirow{2}{*}{$222-224$} & \multirow{2}{*}{80} & 55.51 & 2.33 & 16.18 & 13.65 \\
\hline & & & & 55.42 & 2.30 & 16.13 & 13.62 \\
\hline \multirow{2}{*}{2} & \multirow{2}{*}{$\begin{array}{c}\mathrm{C}_{12} \mathrm{H}_{9} \mathrm{ClN}_{4} \mathrm{O}_{2} \\
(276.68)\end{array}$} & \multirow{2}{*}{$253-254$} & \multirow{2}{*}{75} & 52.09 & 3.28 & 20.25 & 12.81 \\
\hline & & & & 52.00 & 3.26 & 20.18 & 12.77 \\
\hline \multirow{2}{*}{3} & \multirow{2}{*}{$\begin{array}{c}\mathrm{C}_{13} \mathrm{H}_{9} \mathrm{ClN}_{4} \mathrm{O}_{3} \\
(304.69)\end{array}$} & \multirow{2}{*}{$277-278$} & \multirow{2}{*}{70} & 51.25 & 2.98 & 18.39 & 11.64 \\
\hline & & & & 51.30 & 3.00 & 18.45 & 11.60 \\
\hline \multirow{2}{*}{4} & \multirow{2}{*}{$\begin{array}{c}\mathrm{C}_{19} \mathrm{H}_{11} \mathrm{ClN}_{4} \mathrm{O}_{2} \mathrm{~S} \\
(394.83)\end{array}$} & \multirow{2}{*}{ 289-290 } & \multirow{2}{*}{78} & 57.80 & 2.81 & 14.19 & 8.98 \\
\hline & & & & 57.72 & 2.79 & 14.12 & 8.90 \\
\hline \multirow{2}{*}{5} & \multirow{2}{*}{$\begin{array}{c}\mathrm{C}_{13} \mathrm{H}_{7} \mathrm{ClN}_{4} \mathrm{O}_{3} \\
(302.67)\end{array}$} & \multirow{2}{*}{$282-283$} & \multirow{2}{*}{68} & 51.59 & 2.33 & 18.51 & 11.71 \\
\hline & & & & 51.55 & 2.31 & 18.60 & 11.55 \\
\hline \multirow{2}{*}{6} & \multirow{2}{*}{$\begin{array}{c}\mathrm{C}_{22} \mathrm{H}_{14} \mathrm{Cl}_{2} \mathrm{~N}_{4} \mathrm{O}_{3} \mathrm{~S} \\
(485.34)\end{array}$} & & & 54.44 & 2.91 & 11.54 & 14.61 \\
\hline & & $256-258$ & 60 & 54.40 & 2.90 & 11.60 & 14.63 \\
\hline 7 & $\mathrm{C}_{22} \mathrm{H}_{15} \mathrm{ClN}_{4} \mathrm{O}_{3} \mathrm{~S}$ & $236-238$ & 55 & 58.60 & 3.35 & 12.43 & 7.86 \\
\hline 7 & $(450.90)$ & $236-238$ & 53 & 58.55 & 3.33 & 12.40 & 7.80 \\
\hline 8 & $\mathrm{C}_{33} \mathrm{H}_{29} \mathrm{ClN}_{4} \mathrm{O}_{11} \mathrm{~S}$ & $258-257$ & 70 & 54.66 & 4.03 & 7.73 & 4.89 \\
\hline 8 & (725.12) & $258-253$ & 10 & 54.65 & 4.01 & 7.80 & 4.88 \\
\hline 9 & $\mathrm{C}_{19} \mathrm{H}_{13} \mathrm{ClN}_{4} \mathrm{O}_{2}$ & $297-299$ & 73 & 62.56 & 3.59 & 15.36 & 9.72 \\
\hline & $(364.79)$ & & 73 & 62.60 & 3.60 & 15.30 & 9.65 \\
\hline 10 & $\mathrm{C}_{17} \mathrm{H}_{15} \mathrm{ClN}_{4} \mathrm{O}_{2}$ & & 70 & 59.57 & 4.41 & 16.34 & 10.34 \\
\hline 10 & $(342.78)$ & $284-285$ & 70 & 59.55 & 4.40 & 16.33 & 10.29 \\
\hline 11 & $\mathrm{C}_{18} \mathrm{H}_{17} \mathrm{ClN}_{4} \mathrm{O}_{2}$ & $303-304$ & 80 & 60.59 & 4.80 & 15.70 & 9.94 \\
\hline & (356.81) & $303-304$ & 80 & 60.63 & 4.82 & 15.58 & 10.01 \\
\hline 12 & $\mathrm{C}_{16} \mathrm{H}_{10} \mathrm{ClN}_{3} \mathrm{O}_{4}$ & $267-268$ & 60 & 55.91 & 2.93 & 12.23 & 10.31 \\
\hline 12 & (343.72) & $26 /-268$ & 60 & 55.70 & 2.96 & 12.50 & 10.28 \\
\hline 13 & $\mathrm{C}_{14} \mathrm{H}_{7} \mathrm{ClN}_{4} \mathrm{O}_{2} \mathrm{~S}_{3}$ & $213-214$ & 65 & 42.58 & 1.79 & 14.19 & 8.98 \\
\hline & (394.88) & & 65 & 42.60 & 1.80 & 14.15 & 8.90 \\
\hline 14 & $\mathrm{C}_{16} \mathrm{H}_{7} \mathrm{ClN}_{4} \mathrm{O}_{3} \mathrm{~S}_{3}$ & $208-210$ & 53 & 44.19 & 1.62 & 12.88 & 8.15 \\
\hline 14 & $(434.90)$ & $208-210$ & 53 & 44.15 & 1.61 & 12.90 & 8.10 \\
\hline 15 & $\mathrm{C}_{17} \mathrm{H}_{9} \mathrm{ClN}_{4} \mathrm{O}_{3} \mathrm{~S}_{3}$ & $226-228$ & 55 & 45.48 & 2.02 & 12.48 & 7.90 \\
\hline 15 & $(448.93)$ & $2 \angle b-2 \angle 8$ & 35 & 45.52 & 2.00 & 12.53 & 7.87 \\
\hline 16 & $\mathrm{C}_{23} \mathrm{H}_{11} \mathrm{ClN}_{4} \mathrm{O}_{3} \mathrm{~S}_{3}$ & $278-280$ & 78 & 52.82 & 2.12 & 10.71 & 6.78 \\
\hline 16 & $(523.01)$ & $2 / 8-280$ & 78 & 52.80 & 2.14 & 10.80 & 6.77 \\
\hline 17 & $\mathrm{C}_{24} \mathrm{H}_{13} \mathrm{ClN}_{4} \mathrm{O}_{3} \mathrm{~S}_{3}$ & $293-295$ & 80 & 53.68 & 2.44 & 10.43 & 6.60 \\
\hline & (537.03) & & & 53.72 & 2.47 & 10.50 & 6.62 \\
\hline
\end{tabular}


H. H. Sayed et al.: Antimicrobial activity of some synthesized glucopyranosyl-pyrimidine carbonitrile and fused pyrimidine systems, Acta Pharm. 60 (2010) 479-491.

refluxed for $3 \mathrm{~h}$. The reaction mixture was allowed to cool and poured into water. The solid substance was filtered off and crystallized from acetic acid/water to give aryl methylene derivative $\mathbf{1 6}$ or $\mathbf{1 7}$.

Physico-chemical and spectral data of the synthesized compounds are given in Tables I and II.

\section{Antimicrobial activity}

The antibacterial activity of synthesized compounds was tested against Escherichia coli NRRL B-210 (Gram-negative bacterium), Bacillus subtilis NRRL B-543 and Staphylococcus aureus NRRL B-313 (Gram-positive bacteria) using a nutrient agar medium. The

Table II. Spectral data of the new compounds

\begin{tabular}{|c|c|c|c|}
\hline $\begin{array}{l}\text { Compd. } \\
\text { No. }\end{array}$ & $\begin{array}{c}\mathrm{IR} \\
\left(v_{\max }, \mathrm{cm}^{-1}\right)\end{array}$ & ${ }^{1} \mathrm{H} \operatorname{NMR}(\delta, \mathrm{ppm})^{\mathrm{a}}$ & $\operatorname{MS}(m / z, \%)$ \\
\hline 1 & $\begin{array}{l}2221,2225(2 \mathrm{CN}) \\
1685(\mathrm{C}=\mathrm{O})\end{array}$ & $\begin{array}{l}4.9(\mathrm{~s}, 2 \mathrm{H}, \text { oxazolidine }), 7.15(\mathrm{~d}, 2 \mathrm{H}, J=5.8 \\
\mathrm{Hz}, \mathrm{Ar}-\mathrm{H}), 7.62(\mathrm{~d}, 2 \mathrm{H}, J=5.9 \mathrm{~Hz}, \mathrm{Ar}-\mathrm{H})\end{array}$ & $\begin{array}{l}259\left[\mathrm{M}^{+}\right](100), 261 \\
{\left[\mathrm{M}^{+}+2\right](31), 224(14)} \\
195(11), 148(8), 111 \\
(19)\end{array}$ \\
\hline 2 & $\begin{array}{l}3350,3340,3110 \\
\left(2 \mathrm{NH}_{2}, \mathrm{NH}\right), 2217 \\
(\mathrm{CN}), 1686(\mathrm{C}=\mathrm{O})\end{array}$ & $\begin{array}{l}6.50\left(\mathrm{~s}, 2 \mathrm{H}, \mathrm{NH}_{2}, \mathrm{D}_{2} \mathrm{O} \text { exchangeable }\right), 7.2(\mathrm{~d}, \\
2 \mathrm{H}, J=5.8 \mathrm{~Hz}, \mathrm{Ar}-\mathrm{H}), 7.6(\mathrm{~d}, 2 \mathrm{H}, J=5.8 \mathrm{~Hz} \\
\text { Ar- } \mathrm{H}), 7.8\left(\mathrm{~s}, 2 \mathrm{H}, \mathrm{NH}_{2} \text { amide, } \mathrm{D}_{2} \mathrm{O} \text { exchange- }\right. \\
\text { able }), 8.0\left(\mathrm{~s}, 1 \mathrm{H}, \mathrm{NH}, \mathrm{D}_{2} \mathrm{O} \text { exchangeable }\right)\end{array}$ & $\begin{array}{l}276\left[\mathrm{M}^{+}\right](80), 278 \\
{\left[\mathrm{M}^{+}+2\right](26), 241(5)} \\
232(52), 165(10), 150 \\
(25), 126(19), 111(4)\end{array}$ \\
\hline 3 & $\begin{array}{l}3242,3160\left(\mathrm{NH}_{2}\right. \\
2 \mathrm{NH}), 1682,1702 \\
(2 \mathrm{C}=\mathrm{O})\end{array}$ & $\begin{array}{l}6.5(\mathrm{~d}, 2 \mathrm{H}, J=5.3 \mathrm{~Hz}, \mathrm{Ar}-\mathrm{H}), 7.0(\mathrm{~d}, 2 \mathrm{H}, \\
J=5.4 \mathrm{~Hz}, \mathrm{Ar}-\mathrm{H}), 7.57\left(\mathrm{br}, 2 \mathrm{H}, \mathrm{NH}_{2}, \mathrm{D}_{2} \mathrm{O}\right. \\
\text { exhangeable }), 7.91(\mathrm{~s}, 1 \mathrm{H}, \text { pyrimidine } \\
\text { proton), 8.1, } 10.8\left(2 \mathrm{~s}, 2 \mathrm{H}, 2 \mathrm{NH}, \mathrm{D}_{2} \mathrm{O}\right. \\
\text { exchangeable) }\end{array}$ & $\begin{array}{l}304\left[\mathrm{M}^{+}\right](70), 306 \\
{\left[\mathrm{M}^{+}+2\right](20) 260(19)} \\
193(8), 178(28), 126 \\
(11), 111(16)\end{array}$ \\
\hline 4 & $\begin{array}{l}3214,3186(2 \mathrm{NH}) \\
2222(\mathrm{CN}), 1695 \\
(\mathrm{C}=\mathrm{O}), 1256(\mathrm{C}=\mathrm{S})\end{array}$ & $\begin{array}{l}6.40(\mathrm{~d}, 2 \mathrm{H}, J=5.8 \mathrm{~Hz}, \mathrm{Ar}-\mathrm{H}), 6.8(\mathrm{~m}, 2 \mathrm{H}, \\
\mathrm{Ar}-\mathrm{H}), 7.45(\mathrm{~m}, 3 \mathrm{H}, \mathrm{Ar}-\mathrm{H}) 7.7(\mathrm{~d}, 2 \mathrm{H}, \\
J=5.7 \mathrm{~Hz}, \mathrm{Ar}-\mathrm{H}), 8.0,10.6(2 \mathrm{~s}, 2 \mathrm{H}, 2 \mathrm{NH}, \\
\left.\mathrm{D}_{2} \mathrm{O} \text { exchangeable }\right)\end{array}$ & $\begin{array}{l}394\left[\mathrm{M}^{+}\right](60), 396 \\
{\left[\mathrm{M}^{+}+2\right](20), 283(23)} \\
268(14), 126(10), 111 \\
(24)\end{array}$ \\
\hline 5 & $\begin{array}{l}3240,3217,3153 \\
(3 \mathrm{NH}), 2219(\mathrm{CN}), \\
1690,1705(2 \mathrm{C}=\mathrm{O})\end{array}$ & $\begin{array}{l}6.63(\mathrm{~d}, 2 \mathrm{H}, J=5.5 \mathrm{~Hz}, \mathrm{Ar}-\mathrm{H}), 7.2(\mathrm{~d}, 2 \mathrm{H}, \\
J=5.5 \mathrm{~Hz}, \text { Ar-H), } 7.8,10.3,11.0(3 \mathrm{~s}, 3 \mathrm{H}, \\
\text { 3NH, exchangeable) }\end{array}$ & $\begin{array}{l}302\left[\mathrm{M}^{+}\right](49), 304 \\
{\left[\mathrm{M}^{+}+2\right](14), 191(10)} \\
176(5), 126(8), 111 \\
(15)\end{array}$ \\
\hline 6 & $\begin{array}{l}3115(\mathrm{NH}), 2220 \\
(\mathrm{CN}), 1698(\mathrm{C}=\mathrm{O}) \\
1237(\mathrm{C}=\mathrm{S})\end{array}$ & $\begin{array}{l}4.50\left(\mathrm{~s}, 2 \mathrm{H}, \mathrm{CH}_{2}\right), 4.63\left(\mathrm{~s}, 2 \mathrm{H}, \mathrm{CH}_{2}\right), 6.5 \\
(\mathrm{~d}, 2 \mathrm{H}, J=5.8 \mathrm{~Hz}, \mathrm{Ar}-\mathrm{H}), 6.75(\mathrm{~m}, 2 \mathrm{H}, \mathrm{Ar}-\mathrm{H}) \\
7.21(\mathrm{~m}, 3 \mathrm{H}, \mathrm{Ar}-\mathrm{H}), 7.6(\mathrm{~d}, 2 \mathrm{H}, J=5.8 \mathrm{~Hz} \\
\text { Ar- } \mathrm{H}), 7.9\left(\mathrm{~s}, 1 \mathrm{H}, \mathrm{NH}, \mathrm{D}_{2} \mathrm{O} \text { exchangeable }\right)\end{array}$ & $\begin{array}{l}484\left[\mathrm{M}^{+}\right](90), 486 \\
{\left[\mathrm{M}^{+}+2\right](27), 393(30)} \\
358(55), 373(9), 358 \\
(12), 126(23), 111(14)\end{array}$ \\
\hline 7 & $\begin{array}{l}3122(\mathrm{NH}), 2221 \\
(\mathrm{CN}), 1691(\mathrm{C}=\mathrm{O}) \\
1244(\mathrm{C}=\mathrm{S})\end{array}$ & $\begin{array}{l}2.50\left(\mathrm{~m}, 2 \mathrm{H}, \mathrm{CH}_{2} \text {-oxirnyl ring }\right), 2.80(\mathrm{~m}, 1 \mathrm{H}, \\
\mathrm{CH} \text {-oxirnyl ring }), 3.58(\mathrm{~d}, 2 \mathrm{H}, J=3.7 \mathrm{~Hz} \\
\left.\mathrm{CH}_{2}\right), 6.4(\mathrm{~d}, 2 \mathrm{H}, J=5.7 \mathrm{~Hz}, \mathrm{Ar}-\mathrm{H}), 6.64 \\
(\mathrm{~m}, 2 \mathrm{H}, \mathrm{Ar}-\mathrm{H}), 7.01(\mathrm{~m}, 3 \mathrm{H}, \mathrm{Ar}-\mathrm{H}), 7.7 \\
(\mathrm{~d}, 2 \mathrm{H}, J=5.6 \mathrm{~Hz}, \mathrm{Ar}-\mathrm{H}), 7.91(\mathrm{~s}, 1 \mathrm{H}, \mathrm{NH}, \\
\left.\mathrm{D}_{2} \mathrm{O} \text { exchangeable }\right)\end{array}$ & $\begin{array}{l}450\left[\mathrm{M}^{+}\right](27), 439 \\
(45), 424(10), 393(9) \\
226(25), 211(24)\end{array}$ \\
\hline
\end{tabular}


$2.04-2.20\left(4 \mathrm{~s}, 12 \mathrm{H}, 4 \mathrm{COCH}_{3}\right), 3.75(\mathrm{~m}, 2 \mathrm{H}$, H-6', H-6"'), 3.81 (m, 1H, H-5'), 3.98 (m, 2H, $\left.3192(\mathrm{NH}), 2211 \mathrm{H}-4^{\prime}, \mathrm{H}-3^{\prime}\right), 5.49\left(\mathrm{~m}, 1 \mathrm{H}, \mathrm{H}-2^{\prime}\right), 5.70(\mathrm{~d}, 1 \mathrm{H}$,

$8 \quad(\mathrm{CN}), 1718-1667$ $(5 \mathrm{C}=\mathrm{O})$

$\left.J_{1^{\prime}-2^{\prime}}=9.8 \mathrm{~Hz}, \mathrm{H}-1^{\prime}\right), 6.58(\mathrm{~d}, 2 \mathrm{H}, J=5.8 \mathrm{~Hz}$, Ar-H), $6.71(\mathrm{~m}, 2 \mathrm{H}, \mathrm{Ar}-\mathrm{H}), 6.98(\mathrm{~m}, 3 \mathrm{H}, \mathrm{Ar}-\mathrm{H})$ $7.65(\mathrm{~d}, 2 \mathrm{H}, \mathrm{J}=5.9 \mathrm{~Hz}, \mathrm{Ar}-\mathrm{H}), 8.1(\mathrm{~s}, 1 \mathrm{H}, \mathrm{NH}$, $\mathrm{D}_{2} \mathrm{O}$ exchangeable)

5.79 (s, 1H, pyrimidine proton), 6.59 (d, 2H, $J=5.6 \mathrm{~Hz}, \mathrm{Ar}-\mathrm{H}), 6.84$ (m, 2H, Ar-H), 7.10 $725\left[\mathrm{M}^{+}\right](68), 727$ $\left[\mathrm{M}^{+}+2\right](18) 598(23)$, 393 (14), $331(5), 163$ (41), 126 (25), 111 (11)

$3275,3213,3127$

$9(3 \mathrm{NH}), 2218(\mathrm{CN})$, $1689(\mathrm{C}=\mathrm{O})$ $(\mathrm{m}, 3 \mathrm{H}, \mathrm{Ar}-\mathrm{H}), 7.45(\mathrm{~d}, 2 \mathrm{H}, J=5.5 \mathrm{~Hz}, \mathrm{Ar}-\mathrm{H})$ $364\left[\mathrm{M}^{+}\right](38), 366$ $\left[\mathrm{M}^{+}+2\right](9) 287(25)$, 253 (55) 238 (9), 126 $7.9,9.2,10.3\left(3 \mathrm{~s}, 3 \mathrm{H}, 3 \mathrm{NH}, \mathrm{D}_{2} \mathrm{O}\right.$ exchangeable) (46), 111 (23)

1.50-1.96 (m, 8H, aliphatic), 6.89 (d, 2H,

$3280,3219,3121$ $J=5.9 \mathrm{~Hz}, \mathrm{Ar}-\mathrm{H}), 7.2(\mathrm{~d}, 2 \mathrm{H}, J=5.9 \mathrm{~Hz}$,

$10(3 \mathrm{NH}), 2219(\mathrm{CN})$ $1695(\mathrm{C}=\mathrm{O})$ Ar- $\mathrm{H}), 7.93,9.3,10.4$ (3s, 3H, 3NH, pyrimidine, $\mathrm{D}_{2} \mathrm{O}$ exchangeable)

1.30-1.74 (m, 10H, aliphatic), 6.66 (d, 2H,

$3277,3217,3119$

$11(3 \mathrm{NH}), 2215(\mathrm{CN})$ $1679(\mathrm{C}=\mathrm{O})$

$J=5.4 \mathrm{~Hz}, \mathrm{Ar}-\mathrm{H}), 7.31(\mathrm{~d}, 2 \mathrm{H}, J=5.5 \mathrm{~Hz}$,

Ar-H), 8.1, 9.6, 10.1 (3s, 3H, 3NH, pyrimidine, $\mathrm{D}_{2} \mathrm{O}$ exchangeable)

$2218(\mathrm{CN}), 1695$ $1705(2 \mathrm{C}=\mathrm{O})$

1.30 (s, 3H, $\left.\mathrm{CH}_{3}\right), 2.4\left(\mathrm{~s}, 3 \mathrm{H}, \mathrm{OCH}_{3}\right), 6.6$

$(\mathrm{d}, 2 \mathrm{H}, J=5.8 \mathrm{~Hz}, \mathrm{Ar}-\mathrm{H}), 7.3(\mathrm{~d}, 2 \mathrm{H}$, $J=5.8 \mathrm{~Hz}, \mathrm{Ar}-\mathrm{H}$ )

$3225,3150,3125$

(3NH), 1698

13

$(\mathrm{C}=\mathrm{O}), 1260,1255$ $(2 \mathrm{C}=\mathrm{S})$

$6.54(\mathrm{~d}, 2 \mathrm{H}, J=5.6 \mathrm{~Hz}, \mathrm{Ar}-\mathrm{H}), 7.23(\mathrm{~d}, 2 \mathrm{H}$, $J=5.7 \mathrm{~Hz}, \mathrm{Ar}-\mathrm{H}), 9.3,10.7,11.2(3 \mathrm{~s}, 3 \mathrm{H}$, $3 \mathrm{NH}, \mathrm{D}_{2} \mathrm{O}$ exchangeable)

$3210(\mathrm{NH}), 1697,3.30\left(\mathrm{~s}, 2 \mathrm{H}, \mathrm{CH}_{2}\right), 6.48(\mathrm{~d}, 2 \mathrm{H}, J=5.9 \mathrm{~Hz}$,

$141690(2 \mathrm{C}=\mathrm{O}), \quad \mathrm{Ar}-\mathrm{H}), 7.14(\mathrm{~d}, 2 \mathrm{H}, J=5.9 \mathrm{~Hz}, \mathrm{Ar}-\mathrm{H}), 10.7$ $1256,1253(2 \mathrm{C}=\mathrm{S}) \quad\left(\mathrm{s}, 1 \mathrm{H}, \mathrm{NH}, \mathrm{D}_{2} \mathrm{O}\right.$ exchangeable $)$

$3215(\mathrm{NH}), 1700$,

$2.9\left(\mathrm{t}, 2 \mathrm{H}, J=3.3 \mathrm{~Hz}, \mathrm{CH}_{2}\right), 3.12(\mathrm{t}, 2 \mathrm{H}$,

$151694(2 \mathrm{C}=\mathrm{O})$, 1253, $1258(2 \mathrm{C}=\mathrm{S})$

$\left.J=4.2 \mathrm{~Hz}, \mathrm{CH}_{2}\right), 6.78(\mathrm{~d}, 2 \mathrm{H}, J=5.9 \mathrm{~Hz}$, Ar-H), 7.07 (d, 2H, J = 5.9 Hz, Ar-H), 10.8 (s, 1H, NH, $\mathrm{D}_{2} \mathrm{O}$ exchangeable) $6.48(\mathrm{~d}, 2 \mathrm{H}, \mathrm{J}=5.6 \mathrm{~Hz}, \mathrm{Ar}-\mathrm{H}), 6.90$ (m, 3207 (NH), 1715, $1698(2 \mathrm{C}=\mathrm{O})$, $1254,1252(2 \mathrm{C}=\mathrm{S})$ 2H, Ar-H), 7.07 (m, 3H, Ar-H), 7.42 (d, 2H, $J=5.7 \mathrm{~Hz}$, Ar-H), 7.98 (s, 1H, methylene proton), 10.5 (s, $1 \mathrm{H}, \mathrm{NH}, \mathrm{D}_{2} \mathrm{O}$ exchangeable). $3.20\left(\mathrm{~s}, 2 \mathrm{H}, \mathrm{CH}_{2}\right), 6.57(\mathrm{~d}, 2 \mathrm{H}, \mathrm{J}=5.9 \mathrm{~Hz}$, $3217(\mathrm{NH}), 1713, \mathrm{Ar}-\mathrm{H}), 6.90(\mathrm{~m}, 2 \mathrm{H}, \mathrm{Ar}-\mathrm{H}), 7.07(\mathrm{~m}, 3 \mathrm{H}$, $171693(2 \mathrm{C}=\mathrm{O})$, $1255,1249(2 \mathrm{C}=\mathrm{S})$
$342\left[\mathrm{M}^{+}\right](20) 344$ $\left[\mathrm{M}^{+}+2\right](7), 286(10)$, $231(14), 216(8), 126$ (10), $111(25)$

$356\left[\mathrm{M}^{+}\right]$(38), 358 $\left[\mathrm{M}^{+}+2\right](12), 286(45)$, 245 (11), 230 (23), 126 (24), 111 (42)

$343\left[\mathrm{M}^{+}\right]$(65), 345 $\left[\mathrm{M}^{+}+2\right](22), 328(58)$, 232 (43), 175 (54), 168 (12), 111 (23)

$394\left[\mathrm{M}^{+}\right]$(30), 396 $\left[\mathrm{M}^{+}+2\right](9), 378(10)$, 361 (8), 282 (14), 111 (9)

$434\left[\mathrm{M}^{+}\right](30), 436$ $\left[\mathrm{M}^{+}+2\right](12), 322(64)$, 267 (14), 165 (42), 111 (18)

$448\left[\mathrm{M}^{+}\right]$(21), 450 $\left[\mathrm{M}^{+}+2\right](8), 336(12)$, 267 (8), 180 (10), 111 (5)

$523\left[\mathrm{M}^{+}\right](49), 524$

$\left[\mathrm{M}^{+}+2\right](18), 431(54)$, 410 (32), 267 (41), 254 (24), 111 (21)

$537\left[\mathrm{M}^{+}\right](47), 538$ $\left[\mathrm{M}^{+}+2\right](15), 445$ (64), 424 (25), 268 (14), 111 (21)

a The solvent for compounds $1,2,3,5,7,9,14$ is $\mathrm{CDCl}_{3}$ and for compounds $4,6,10,11,12,13,15,16,17,18$ is DMSO- $d_{6}$. 
H. H. Sayed et al:: Antimicrobial activity of some synthesized glucopyranosyl-pyrimidine carbonitrile and fused pyrimidine systems, Acta Pharm. 60 (2010) 479-491.

antifungal activity of the compounds was tested against Candida albicans NRRL Y-477 using the Sabouraud dextrose agar medium.

Agar diffusion medium. - Nine compounds were screened in vitro for their antimicrobial activity by the agar diffusion method (14). A suspension of organisms was added to a sterile nutrient agar medium at $45^{\circ} \mathrm{C}$ and the mixture was transferred to a sterile Petri dish and allowed to solidify. Holes of $10 \mathrm{~mm}$ in diameter were made using a cork borer and filled with the solution of synthesized compounds $\left(100 \mu \mathrm{g} \mathrm{mL} \mathrm{m}^{-1}\right)$. A hole filled with DMSO was used as control. The plates were left for 1 hour at room temperature as a period of pre-incubation. The plates were then incubated at $37^{\circ} \mathrm{C}$ for 24 hours and observed for antibacterial activity. Diameters of the zone of inhibition were measured and compared with that of the standard. Ciprofloxacin $(50 \mu \mathrm{g} \mathrm{mL}-1)$ and ketoconazole $(50 \mu \mathrm{g}$ $\mathrm{mL}^{-1}$ ) were used as standards for antibacterial and antifungal activity, respectively. The observed zones of inhibition are presented in Table III.

Minimum inhibitory concentration. - Minimum inhibitory concentration (MIC) of the test compounds was determined by the agar streak dilution method. Stock solutions of synthesized compounds were made using DMSO as a solvent $\left(68 \mathrm{mg} \mathrm{mL}^{-1}\right)$. From this stock solution, a series of concentrations was prepared $(0.17,0.34,0.68,0.85$ and $1.7 \mathrm{mg}$ $\mathrm{mL}^{-1}$ ) and mixed with known quantities of molten sterile agar medium aseptically. About $20 \mathrm{~mL}$ of the medium containing the tested compound was dispensed into a sterile Petri dish. Then, the medium was allowed to solidify. Microorganisms were then streaked one by one on the agar plates aseptically. After streaking, all the plates were incubated at $37^{\circ} \mathrm{C}$ for $24-48 \mathrm{~h}$ for antibacterial and antifungal activity, respectively. The lowest concentration of the synthesized compound that inhibits the growth of the given bacterium/fungus was considered as the minimum inhibitory concentration (MIC) of the test compounds. The MIC values are tabulated in Table IV.

\section{RESULTS AND DISCUSSION}

\section{Chemistry}

4-Oxo-oxazolidin-2-ylidene malononitrile derivative 1 was prepared via condensation of malononitrile with $p$-chlorophenylisocyanate in alkaline medium, followed by addition of ethyl chloroacetate. Ammoniation of compound 1 afforded 3-amino-5-(4-chlorophenylamino)-4-cyanofuran-2-carboxamide (2) according to the mechanism given in Scheme 1. Compound 2 was used to synthesize some fused heterocyclic derivatives. Thus, heating of compound 2 with an aliphatic acid, namely formic acid, resulted in the formation of 3,4-dihydrofuro[3,2-d]pyrimidine 3. Also, when reacted with phenylisothiocyanate in refluxing acetic acid it afforded the corresponding 1,2,3,4-tetrahydrofuro[3,2- $d$ ] pyrimidine-7-carbonitrile 4 (Scheme 2 ). All the synthesized compounds were characterized by their physical, chemical and spectral data (Tables I and II). IR spectra of compound 3 showed the presence of absorption bands at 1682 and $1702 \mathrm{~cm}^{-1}(2 \mathrm{C}=\mathrm{O})$ and the absence of a characteristic band $(\mathrm{CN})$ due to hydrolysis of the cyano group; the MS gave the molecular ion peak at $m / z(\%)=394(60)$. 
H. H. Sayed et al.: Antimicrobial activity of some synthesized glucopyranosyl-pyrimidine carbonitrile and fused pyrimidine systems, Acta Pharm. 60 (2010) 479-491.

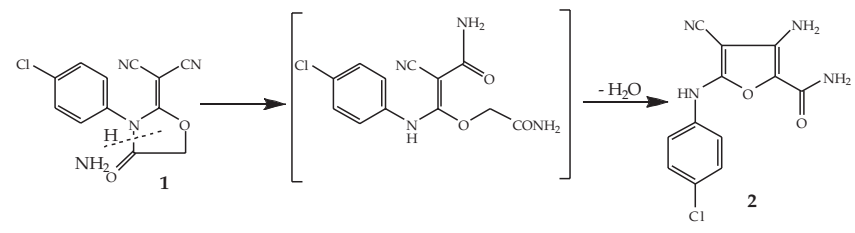

Scheme 1

Furthermore, 4-cyanofuran-2-carboxamide 2 reacted with ethylchloroformate in refluxing acetic acid to yield 2,4-dioxofuro[3,2-d]pyrimidine-7-carbonitrile 5. Beside correct values of elemental analyses, the spectral data for 5 are in agreement with the assigned structure (Scheme 2).
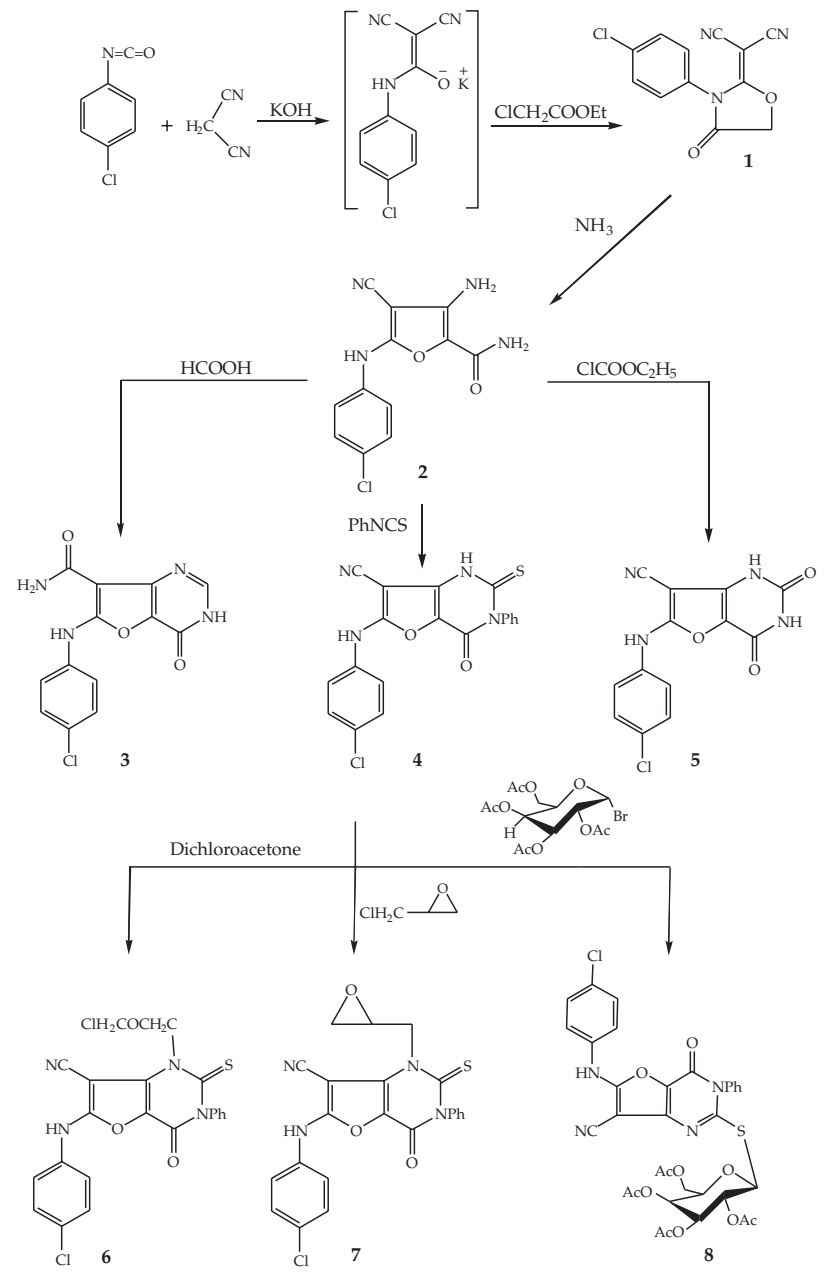

Scheme 2 
In addition, alkylation of compound 4 with dichloroacetone or epichlorohydrine in the presence of sodium hydride afforded the corresponding $N$-alkyl pyrimidine derivatives 6 and 7, respectively. On the other hand, alkylation of 4 with 2,3,4,6-tetra-O-acetyl $-\alpha$-D-glucosyl bromide in acetone and in the presence of aqueous potassium hydroxide afforded the corresponding S-glucosides 8 in good yield (Scheme 2). Attachment of the glucosyl residues to the sulfur atom rather than to the nitrogen atom was supported by the value of the chemical shift of the anomeric protons which should other-wise appear at a lower field. The anomeric proton of $\beta-N$-glucosides having an adjacent $C=S$ was reported (15) to appear at a higher chemical shift $(\delta 6.90-7.20 \mathrm{ppm})$ due to the anisotropic deshielding effect of the $\mathrm{C}=\mathrm{S}$. The structure of $\mathbf{8}$ was confirmed by elemental analysis and spectral data. The IR spectrum showed absorption bands at 3192, 2211 and 1718-1667 $\mathrm{cm}^{-1}$ due to the presence of $\mathrm{NH}, \mathrm{CN}$ and $\mathrm{C}=\mathrm{O}$ groups, respectively. The ${ }^{1} \mathrm{H}$ NMR spectrum showed the anomeric proton of the glucose moiety as a doublet at $\delta 5.70 \mathrm{ppm}$ with a coupling constant $J_{1^{\prime}-2^{\prime}}=9.8 \mathrm{~Hz}$, indicating that $\mathrm{H}-1^{\prime}$ is trance-dioxal to $\mathrm{H}-2^{\prime}$. The other six glucose protons resonated at $\delta 3.75-5.49 \mathrm{ppm}$ and four acetyl groups appeared as four singlet peaks at $\delta 2.04-2.20 \mathrm{ppm}$. In addition, its $\mathrm{C}, \mathrm{H}, \mathrm{N}$ analysis data revealed the molecular formula $\mathrm{C}_{33} \mathrm{H}_{29} \mathrm{ClN}_{4} \mathrm{O}_{11} \mathrm{~S}$.

When compound 2 was allowed to react with benzaldehyde, it afforded 6-(4-chloro-phenylamino)-4-oxo-2-phenyl-1,2,3,4-tetrahydrofuro[3,2-d]pyrimidine-7-carbonitrile (9) in good yield (Scheme 3). Also, compound 2 reacted with cyclopentanone or cyclohexanone in refluxing acetic acid and sodium acetate to afford the corresponding pyrimidine derivatives 10 and 11, respectively. On the other hand, when compound 2 was treated with acetic anhydride, it afforded the corresponding furo[3,2- $d][1,3]$ oxazine derivative 12 (Scheme 3). The ${ }^{1} \mathrm{H}$ NMR spectrum of compound 12, for example, showed a singlet at $\delta 1.30 \mathrm{ppm}$, which supported the methyl protons and signal at $\delta 2.4 \mathrm{ppm}$ for the methoxy group, supporting the acetylation of $\mathrm{NH}$ group. The MS gave the molecular ion peak at $m / z(\%)=343(65)$.

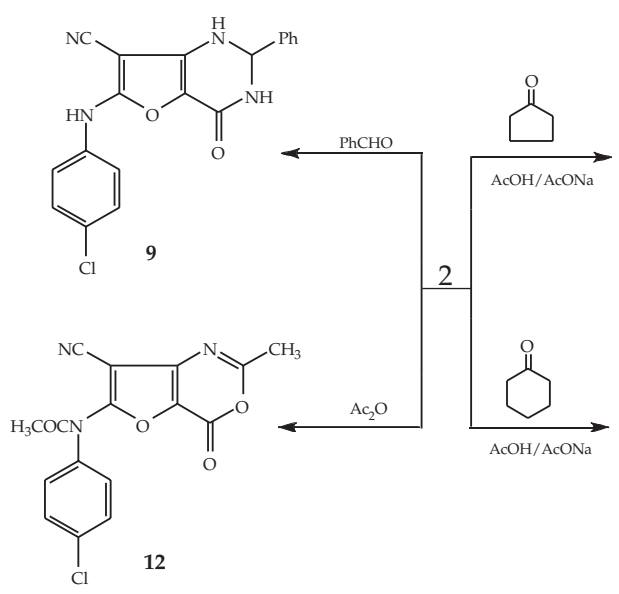<smiles>N#Cc1c(Nc2ccc(Cl)cc2)oc2c1NC1(CCCC1)NC2=O</smiles>

Scheme 3 
A very interesting reaction occurred when compound 2 was allowed to react with carbon disulfide in dry pyridine to produce 1-(4-chlorophenyl)-4-imino-2,6-dithioxo-1,4, 6,7-tetrahydro-pyrimido[5,4-b]furo[5,4-d]-1,3-thiazin-8-one (13) (Scheme 4). The structure of compound 13 was confirmed from its spectral data. On the other hand, when treated with aliphatic acids, namely chloroacetic acid or chloropropionic acid compound 13 afforded polycyclic fused compounds 14 and 15, respectively. Beside the values in elemental analyses and spectral data, the latter compounds were confirmed chemically via condensation with benzaldehyde to give the corresponding aryl methylene compounds 16 and 17, respectively (Scheme 4).

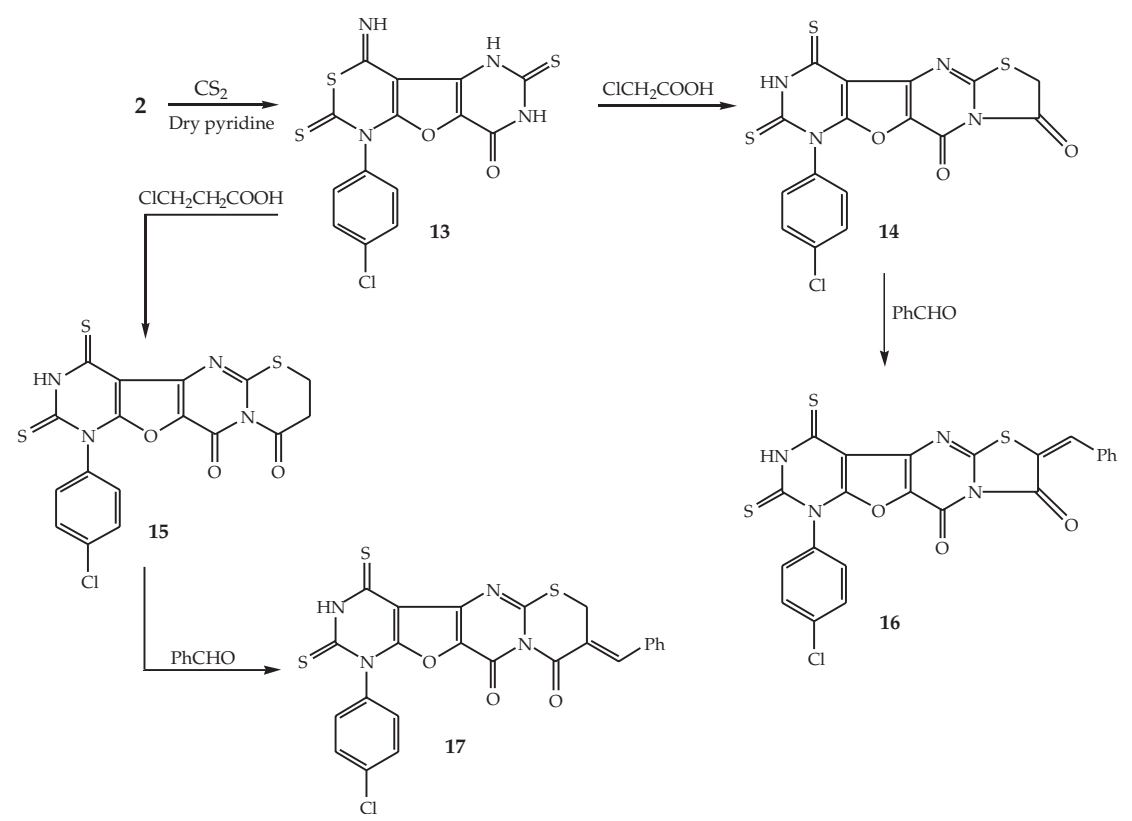

Scheme 4

\section{Antimicrobial activity}

The antimicrobial results as the average diameter of inhibition zones, expressed in $\mathrm{mm}$ are given in Table III. It is evident that all tested compounds display activity against Bacillus subtilis, Staphylococcus aureus and Escherichia coli while only compounds 6, 9, 11, 17 and 18 were active against Candida albicans as well. Compounds 5, 6, and 8 were the most active ones against all the listed bacteria. Mean inhibition zones and minimal inhibitory concentrations of these compounds ranged from 20 to $30 \mathrm{~mm}$ and from 0.17 to $0.68 \mathrm{mg} \mathrm{mL}^{-1}$, respectively. Compounds 6 and 8 also exhibited activity against the fungus $C$. albicans. All tested compounds showed activity lower than that of the standard drugs (ciprofloxacin and ketaconazole). 
H. H. Sayed et al.: Antimicrobial activity of some synthesized glucopyranosyl-pyrimidine carbonitrile and fused pyrimidine systems, Acta Pharm. 60 (2010) 479-491.

Table III. Inhibition zones of the newly synthesized compounds

\begin{tabular}{ccccc}
\hline & \multicolumn{4}{c}{ Inhibition zone } \\
\cline { 2 - 5 } $\begin{array}{c}\text { Compd. } \\
\text { No. }\end{array}$ & Gram positive bacteria & $\begin{array}{c}\text { Gram negative } \\
\text { bacteria }\end{array}$ & Fungus \\
\cline { 2 - 5 } & Bacillus subtilis & Staphylococcus aureus & Escherichia coli & Candida albicans \\
\hline DMSO(solvent) & - & - & - & - \\
$\mathbf{2}^{\mathbf{a}}$ & 14 & 21 & 21 & - \\
$\mathbf{3}^{\mathbf{a}}$ & 13 & 19 & 16 & - \\
$\mathbf{5}^{\mathbf{a}}$ & 23 & 25 & 30 & 17 \\
$\mathbf{6}^{\mathbf{a}}$ & 25 & 25 & 20 & 14 \\
$\mathbf{8}^{\mathbf{a}}$ & 20 & 25 & 24 & - \\
$\mathbf{9}^{\mathbf{a}}$ & 14 & 18 & 19 & 16 \\
$\mathbf{1 0}^{\mathbf{a}}$ & 19 & 24 & 20 & 16 \\
$\mathbf{1 6}^{\mathbf{a}}$ & 16 & 20 & 14 & 20 \\
$\mathbf{1 7}^{\mathbf{a}}$ & 17 & 22 & 21 & - \\
Ciprofloxacin $^{\mathrm{b}}$ & 23 & 23 & 25 & 23 \\
Ketaconazole $^{\mathbf{b}}$ & - & - & - & - \\
\hline
\end{tabular}

a $100 \mu \mathrm{g} \mathrm{mL}^{-1}$

b $50 \mu \mathrm{g} \mathrm{mL}^{-1}$

Table IV. MIC of the newly synthesized compounds

\begin{tabular}{|c|c|c|c|c|}
\hline \multirow{3}{*}{$\begin{array}{l}\text { Compd. } \\
\text { No. }\end{array}$} & \multicolumn{4}{|c|}{ Minimum inhibitory concentration $\left(\mathrm{mg} \mathrm{mL}^{-1}\right)$} \\
\hline & \multicolumn{2}{|c|}{ Gram positive bacteria } & \multirow{2}{*}{$\begin{array}{c}\text { Gram negative bacteria } \\
\text { Escherichia coli }\end{array}$} & \multirow{2}{*}{$\begin{array}{c}\text { Fungus } \\
\text { Candida albicans }\end{array}$} \\
\hline & Bacillus subtilis & Staphylococcus aureus & & \\
\hline 5 & 0.34 & 0.34 & 0.17 & 1.7 \\
\hline 6 & 0.34 & 0.34 & 0.68 & 0.85 \\
\hline 8 & 0.68 & 0.34 & 0.34 & 1.7 \\
\hline 10 & 0.68 & 0.34 & 0.68 & 0.85 \\
\hline 17 & 0.85 & 0.68 & 0.68 & 0.68 \\
\hline $\begin{array}{l}\text { Ciprofloxacin } \\
\left(\mu \mathrm{g} \mathrm{mL} L^{-1}\right)\end{array}$ & 0.12 & 0.15 & 0.01 & - \\
\hline $\begin{array}{l}\text { Ketaconazole } \\
\left(\mu \mathrm{g} \mathrm{mL}^{-1}\right)\end{array}$ & - & - & - & 0.03 \\
\hline
\end{tabular}




\section{CONCLUSIONS}

Screening data of the prepared compounds show promising antibacterial and antifungal activity. Compounds 8, 10 and 16 showed significant antibacterial activity due to the presence of the sugar moiety and addition of cycloalkane or thiazole to the furanopyrimidine ring. Efficacy of compound 6 is probably due to addition of chloroacetone to the furopyrimidine ring.

\section{REFERENCES}

1. H. H. Sayed, A. H. Mostafa, N. M. Yousif, M. G. Assy and M. A. Abd El-Halim, Synthesis and reactions of some novel mercaptopyrimidine derivatives for biological evaluation, Phosphorus Sulfur Silicon 183 (2008) 2318-2329; DOI: 10.1080/10426500801963590.

2. H. H. Sayed, A. H. Shamroukh and A. E. Rashad, Synthesis and biological evaluation of some pyrimidine, pyrido[2,1-b][1.3]thiazine and thiazolo[3,2-a]pyrimidine derivatives, Acta Pharm. 56 (2006) 231-244.

3. S. A. Said, A. E. Amr, N. M. Sabry and M. M. Abdalla, Analgesic, anticonvulsant and antiinflammatory activities of some synthesized benzodiazipine, triazolopyrimidine and bis-imide derivatives, Eur. J. Med. Chem. 44 (2009) 4787-4792; DOI: 10.1016/j.ejmech.2009.07.013.

4. S. F. Mohamed, E. M. Flefel, A. E. Amr and D. N. Abd El-Shafy, Anti-HSV-1 activity and mechanism of action of some new synthesized substituted pyrimidine, thiopyrimidine and thiazolopyrimidine derivatives, Eur. J. Med. Chem. 45 (2010) 1494-1501; DOI: 10.1016/j.ejmech.2009.12. 057

5. M. S. El-Gaby, S. G. Abdel-Hamid, M. M. Ghorab and S. M. El-Sayed, Synthesis and anticancer activity in vitro of some new pyrimidines, Acta Pharm. 49 (1999) 149-158.

6. A. B. A. El-Gazzar, H. N. Hafez and S. A. Hebat-Allah, S- and C-nucleosidoquinazoline as new nucleoside analogs with potential analgesic and anti-inflammatory activity, Eur. Med. Chem. 44 (2009) 4249-4258; DOI: 10.1016/j.ejmech.2009.05.025.

7. K. Leach, S. M. Swaney, J. R. Colca, W. G. McDonald, J. R. Blinn, L. M. Thamasco, R. C. Gadwood, D. Shinbarger, L. Xiong and A. S. Mankim, The site of action of oxazolidinone antibiotics in living bacteria and human mitochondria S, Mol. Cell 26 (2007) 393-402; DOI: 10.1016/j.molcel. 2007.04.005.

8. A. E. Amr, A. A. Nehad and M. M. Abdalla, Synthesis and antiandrogenic activity of some new 3-substituted androstano[17,16-c]-5'-aryl-pyrazoline and their derivatives, Bioorg. Med. Chem. 14 (2006) 373-380; DOI: 10.1016/j.bmc.2005.08.024.

9. A. E. Amr and M. M. Abdalla, Anti-inflammatory profile of some synthesized heterocyclic pyridine and pyridine derivatives fused with steroidal structure, Bioorg. Med. Chem. 14 (2006) 4341-4352; DOI: $10.1016 /$ j.bmc.2006.02.045.

10. S. A. Hebat-Allah, W. A. El Sayed and F. N. Fathy, Synthesis and antitumor activity of new dihydropyridine thioglycosides and their corresponding dehydrogenated forms, Eur. Med. Chem. 45 (2010) 973-982; DOI: 10.1016/j.ejmech.2009.11.039.

11. A. E. Amr, H. H. Sayed and M. M. Abdalla, Synthesis and reactions of some new substituted pyridine and pyrimidine derivatives as analgesic, anticonvulsant and antiparkinsonian agents, Arch. Pharm. Chem. Life Sci. 338 (2005) 433-440; DOI: 10.1002/ardp.200500982. 
H. H. Sayed et al.: Antimicrobial activity of some synthesized glucopyranosyl-pyrimidine carbonitrile and fused pyrimidine systems, Acta Pharm. 60 (2010) 479-491.

12. H. N. Hafez, S. A. Hebat-Allah and A. B. A. El-Gazzar, Synthesis and evaluation of analgesic, anti-inflammatory and ulcerogenic activities of some triazolo- and 2-pyrazolylpyrido[2,3-d]-pyrimidines, Acta Pharm. 58 (2008); 359-378; DOI: 10.2478/v10007-008-0024-1.

13. M. H. Abo-Ghalia and A. E. Amr, Synthesis and investigation of a new cyclo-(N ${ }^{\alpha}$-dipicolinoyl) pentapeptide of a breast and CNS cytotoxic activity and an ionophoric specifity, Amino Acids 26 (2004) 283-289; DOI: 10.1007/s00726-003-0042-8.

14. S. R. Jain and A. Kar, The antibacterial activity of some essential oils and their combinations. Planta Med. 20 (1971) 118-123; DOI: 10.1055/s-0028-1099675.

15. K. Mansour, Y. A. Ibrahim and N. S. A. M. Khalil, Nucleos. Nucleot. Nucl. 18 (1999) 2265-2283; DOI: $10.1080 / 07328319908044880$.

$S A \check{Z} E T A K$

\section{Antimikrobno djelovanje nekih glukopiranozil-pirimidin karbonitrila i fuzioniranih pirimidinskih sustava}

HAYAM H. SAYED, HEBAT-ALLAH S. ABBAS, EMAN M. H. MORSI, ABD EL-GALIL E. AMR i NAYERA A. M. ABDELWAHAD

3-Amino-5-(4-klorfenilamino)-4-cijanofuran-2-karboksamid (2) upotrebljen je kao ključni spoj za pripravu različitih furo-pirimidina 3-9 i spiro-cikloalkan furopirimidina 10 i 11. Fuzionirani heterociklički spojevi 13-17 pripravljeni su također polazeći iz spoja 2. Sintetizirani spojevi ispitani su na antimikrobno djelovanje.

Ključne riječi: pirimidin, glukopiranozil-pirimidin, oksazin, antimikrobno djelovanje

Photochemistry Department, National Research Centre, 12622 Dokki, Cairo, Egypt

Department of Pharmaceutical Chemistry, College of Pharmacy, King Saud University, Riyadh 11451 Saudi Arabia

Chemistry of Natural and Microbial Product Department, National Research Centre, 12622 Dokki, Cairo Egypt 